background. Another feature that shows up in this plot (Figure 8 of the LaBonte and Howard paper) is a one-cycle-per-hemisphere torsional wave which is characterized by a faster rotation at high latitudes near solar minimum and a faster rotation at low latitudes near solar maximum. The amplitude is roughly that of the traveling torsional wave, i.e. $\sim 5 \mathrm{~m} \mathrm{~s}^{-1}$. The high latitude portion of this pattern was found earlier by Livingston and Duvall (1979).

There is evidence for a $1 / 2$ cycle-per-hemisphere oscillation in a comparison of equatorial rotation rates determined from north and south data separately (Howard et al. 1983). The amplitude is comparable to those of the two modes mentioned above.

The cyclic variation of the rotation rate of the Sun determined from sunspot and Doppler data discussed above is essentially another mode of torsional oscillation. In this case, the amplitude is about $200 \mathrm{~ms}^{-1}$, which is much larger than the other modes.

\title{
References
}

Abarbanell, C. and Wöhl, H. 1981, Solar Phys., 70, 197.

Arevalo, M.J. et al. 1982, Astron. and Astrophys., 111, 266.

Balthasar, H. and Schössler, M. 1982, Solar Phys., 87, 23.

Balthasar, H. 1983, Solar Phys., 84, 371.

Cram, L.E., Durney, B.R. and Guenther, D.B. 1983, Ap. J., 267, 442.

Dicke, R.H. 1982, Solar Phys., 78, 3.

Duvall, T.L., Jr. 1982, Solar Phys, 76, 137.

Gilman, P.A. and Howard, R. 1984, Ap. J., 283, 285.

Godoli, G. and Mazzucconi, F. 1983, Solar Phys., 83, 339.

Howard, R. et al. 1983, Solar Phys., 87, 195.

Howard, R., Gilman, P.A. and Gilman, P.I. 1984, Ap. J., 283, 373.

Koch, A., Wohl, H. and Schröter, E.H. 1981, Solar Phys., 71, 295.

Kopecky, M. 1982, Bull. Astron. Inst. Czech., 33, 285.

Kubicela, A. and Karabin, M. 1983, Solar Phys., 84, 389.

Kuveler, G. and Wöhl, H. 1983, Astron. and Astrophys., 123, 29.

LaBonte, B.J. and Howard, R. 1982, Solar Phys., 75, 161.

Landman, D.A. and Takushi, J.T. 1981, Solar Phys., 73, 379.

Libbrecht, K. 1984, Ph.D. Thesis: Princeton University.

Livingston, W. and Duvall, T.L., Jr. 1979, Solar Phys., 61, 219.

Lustig, G. 1982, Astron. and Astrophys., 106, 151.

Lustig, G. 1983, Astron. and Astrophys., 125, 355.

Newton, H.W. and Nunn, M.L. 1951, M.N.R.A.S., 111, 413.

Perez-Garde, M. et al. 1981, Astron. and Astrophys., 93, 67.

Rovithis, P. 1982, Astrophysics and Space Science, 88, 5.

Snider, J.L. 1983, solar Phys., 84, 377.

Snodgrass, H.B. 1983, Ap. J., in press.

Ternullo, M., Zappala, R.A., and Zuccarello, F. 1981, Solar Phys., 74, 111.

Tuominen, J., Tuominen, I., and Kyrölöinen, J. 1983, M.N.R.A.S., 205, 69.

Yallop, B.D., Hohenkerk, C., Murdin, L. and Clark, D.H. 1982, Quart. J.R.A.S., 23, 213.

\section{SOLAR GRANULATION}

\section{(R. Muller)}

During the period 1981-1984, major developments have been made in the analysis of the shape and shifts of photospheric lines used as a diagnostic of the convection in the outer layers of the Sun. The first three-dimensional numerical simulation of the solar granulation has been developed, and it is able to reproduce with some success most of the observed morphological and spectroscopic properties of granulation. It is becoming clear that the properties of the solar granulation are variable over the solar cycle. Many questions remain to be solved; further progress will rely heavily on expected improvements of the spatial resolution of the observations and the availability of larger computers. 


\section{Morphological structure of the solar granulation.}

Wittmann (1981), analysing high resolution Spektro-Stratoskop photographs (Mehltretter, 1978), confirms several accepted values like the fractional area of granules (47.1\%), the corrected contrast (22\%), and the asymmetry of intensity histograms. However, he finds a somewhat larger granular size than usually accepted, and a shorter lifetime $(5.8 \mathrm{~min})$.

It appears that granules are more evenly spaced than random points (Lawrence, 1983) and form bright chains, with contrast exceeding the mean contrast of granulation by several percent and of longer lifetime (Perfinenko, 1981). Confirming and extending the last result, Oda (1984) finds that the bright and exploding granules tend to form chains, defining a cellular pattern comparable in size to that of the mesogranulation. Isophotal contour maps of a single granule are presented by Bray and Loughhead (1984).

Recent measurements of the granulation contrast are in reasonable agreement around $5500 \mathrm{~A}$, but are very uncertain in the blue and the red, so that they do not allow comparison with models (Bray, 1982).

\section{Statistical analysis of brightness fluctuations.}

New evaluations of rms brightness fluctuations $\left(\Delta \mathrm{I}_{\text {rms }}\right)$, corrected for instrumental and atmospheric blurring fall near the lower limit of the range $10-20 \%$ from previous evaluations (Schmidt et al. 1981, solar eclipse observation at Izana; Durrant et al., 1983, Spektro-Stratoskop pictures). As these new values are not safer than the previous ones, the true $\Delta \mathrm{I}_{\text {rms }}$ of the solar granulation remains dubious. It is confirmed that the change of rms fluctuation from the centre of the disk to $\mu=0.3$ is small. Fitting the observed spread function of Deubner and Mattig (1975) by the sum of two Lorentzians, rather than by two Gaussians, Nordlung (1984a) re-evaluated the $\Delta \mathrm{I}_{\text {rms }}$ of the solar granulation which is thus increased from $12.7 \%$ (Deubner and Mattig, 1975) to about $20 \%$ at 6070 Angstrom. This value is in good agreement with the $\Delta \mathrm{I}$ rms of the Nordlung's tri-dimensional numerical model (Nordlung, 1982) of $20-25 \%$ at 6000 Angstrom.

Bi-dimensional power spectra obtained by Schmidt et al. (1981) and Durrant et al. (1983) are in good agreement with previously published single peak power spectra, while the bi-dimensional power spectrum, derived from one dimensional scans by Wittmann (1981), exhibits a multi-component peak; similar peak splitting was already reported in the past by a few authors but its existence as a property of the solar granulation remains doubtful.

The correct formulation of statistical analysis in the general case of a tilted image is derived by Wiesmeier and Durrant (1981) both for uni- and bi-dimensional observations; restoration by the point spread function is included in the formulation. V. der Lühe (1981) demonstrated that Optical Fourier Transformation, if carefully handled, may be a reliable alternative to the usual numerical procedures for deriving the power spectrum of the solar granulation. Ricort et al. (1982) performed estimations of Fried's parameter $r_{o}$, simultaneously using the same telescope, from the observed solar granulation contrast and from the variance of angle-of- arrival fluctuations; $r_{o}$ obtained by both methods are well correlated.

Fluctuations of the limb position, due to brightness fluctuations of the granular pattern, do not exceed a few thousandths of a second of arc (Lites, 1982).

\section{Empirical models of temperature and velocity.}

Temperature models. Durrant et al. (1981) have shown that if the "geometrical smoothing" toward the limb is taken into account, recent empirical temperature models of the solar granulation, in which the temperature fluctuation is rapidly vanishing in the lower photosphere, are able to reproduce the centre-to-limb variation of the continuum intensity, as well as the intensity fluctuations in spectral lines, observed with the Spektro-Stratoskop. Karpinsky (1981) reports on measurements of the horizontal gradient of brightness of granules of 1.5 to $2.0 \mathrm{~K} / \mathrm{km}$, the peak values reaching $2.7 \mathrm{~K} / \mathrm{km}$; the values can be as high as 5.0 to $10.0 \mathrm{~K} / \mathrm{km}$ when corrected for distortions.

Velocity models. The steepness of the decrease with height in the photosphere of the rms vertical velocity field associated with the solar granulation is a much debated question (see, for example, the flat gradient model of Durrant et al., 1979 and the steep gradient model of Keil, 1980). It seems that the discrepancy is a result of the different procedures used to separate the convective and oscillatory components. The results of Bässgen and Deubner (1982) who analyse a high resolution time series of spectra obtained at Sacramento Peak Observatory, and use the resulting $k-\omega$ diagram to separate convective and oscillating powers, favor the flat gradient model. The coherence of the vertical convective velocities is maintained at least up to $300 \mathrm{~km}$ in the photosphere (Durrant 
and Nesis, 1982; Pravdjuk, 1982), confirming previous results. The penetration of convective cells to such high levels also favors a flat gradient model. It is found by Durrant and Nesis that the velocity coherence of the structures of sizes in the range $2-6 \operatorname{arcsec}$ is maintained high up in to the photosphere while it is lost more rapidly for smaller structures.

It is confirmed that the coherence between continuum intensities and vertical velocities is restricted to the lower photosphere (Durrant and Nesis, 1982; Nesis et al., 1983). The horizontal velocity patterns are not coherent with the vertical velocity, or with the brightness pattern, indicating lack of horizontal heat transport. It is not possible to conclude how the horizontal velocity and its variation with the beight in the atmosphere is related to the structure of granules (Nesis et al., 1983).

An analysis of the Doppler shift fluctuations in the profile of the strong $\mathrm{Na}_{\mathrm{I}} \mathrm{D}_{1}$ line, instead of the more usual analysis of fluctuations in the core of lines of different strengths, failed to provide new information about the structure of the velocity field in the photosphere (Edmonds and Jin-Chung Hsu, 1983).

\section{Shift and asymmetry of photospheric line profiles.}

Spatially unresolved photospheric line profiles are asymmetric (C-shape of the line bisector) and blueshifted; these properties are signatures of the presence of convection and are used to get information about the vertical and horizontal structure of the photosphere; but this is not a trivial problem.

Line shift and asymmetry as tests for convection models. Kaisig and Durrant (1982) investigate in detail the information content of the shifts of mean line profiles with the aid of a perturbation analysis and a two-stream model. They demonstrate that the characteristic C-shape of mean solar line profiles is due not to the larger velocity amplitude of the downflow as proposed by Dravins et al. (1981), but to a complex opacity effect. The analysis of shifts of photospheric lines does not allow separation of the depth dependence of velocity, $\delta \mathrm{v}(\mathrm{h})$, and temperature, $\delta \mathrm{T}(\mathrm{h})$, fluctuations, but it is useful as tests of convective models (Kaisig and Durrant, 1982, Kaisig and Schröter, 1983; Kostick, 1983).

Methods based on the analysis of the third central moment $\mathrm{M}_{3}$ of the line profiles (Marmolino and Severino, 1981) and on a linear analysis of the bisector (Buonaura and Caccin, 1982) have been developed to retrieve $\delta \mathrm{v}(\mathrm{h})$. The main limitation of both methods is that they are applicable only to data with infinite resolution. Pierce (1984) shows that comparing an observed Fraunhofer line to a fitted gaussian yields more information on shape and asymmetry of the solar line than the simple bisector method.

Line asymmetry and blue-shift-parameters dependence. Balthasar (1984) finds that lines with cores formed in higher layers show larger asymmetries. Analysis of lines of ionized elements (Fe II) confirms the weak increase of the blue-shift with increasing excitation potential found for neutral elements (Dravins and Larsson, 1983). These authors also report that lines formed in shorter wavelength regions are more blue-shifted than lines in the red. In their investigation, Dravins and Larsson used the Kitt Peak Wavelength Table by Pierce and Breckinridge (1973), while Balthasar used observations obtained with the Fourier-Transform-Spectrometer (FTS) at the Mac Math Telescope of the Kitt Peak National Observatory (KPNO).

Some authors find that line asymmetry changes with time (Roca-Cortes et al., 1983, but the line KI 7699 investigated is formed high in the photosphere and is more sensitive to oscillations than to convection), while others do not (Cavallini et al., 1982).

Accurate measurements of the shape of $\mathrm{Fe} I 6301.5$, performed with a Fabry-Perot spectrometer, agree with previous measurements (Cavallini et al., 1982). The shape of the KI 7699 line profile is also confirmed by new measurements (Roca-Cortes et al., 1983.

Limb effect. The blue-shift progressively decreases as one moves away from the disk centre and may even become a supergravitational redshift at the extreme limb: this is known as the limb effect. Balthasar (1984) confirms the existence of supergravitational red-shifts at the limb for many photospheric lines; in addition he finds that the red-shifts for lines from ionized elements are larger than those from neutral elements. Brandt and Schröter (1982), analysing spectra with iodine reference lines obtained at Locarno Observatory, confirm earlier findings that the limb effect is a combination of shifts of the line centre and the centre-to-limb variation of the shape; at the limb the asymmetry disappears. The relative blue-shift of many lines at $\cos \theta=0.8$ compared to the disk center is confirmed by Brandt and Shröter (1982) and by Balthasar (1984). 
Latitude variation. It appears that there is a significant difference between the limb-effect curves along the polar and equatorial diameters for $\cos \theta \leq 0.4$ and a rather strong indication of a latitude dependence of the line bisector C-shape (Brandt and Schröter, 1982). These authors come to the conclusion that this latitude dependence may account for the so-called "ears" observed by Howard et al. (1980).

Line asymmetry and blue-shift in plages. The C-shape of line bisector is flatter in plages than in the quiet Sun (Livingston, 1982; Kaisig and Schröter, 1983; Brandt and Schröter, 1984; Cavallini et al., 1984). There is presently disagreement about the relative shift of the line bisector in plage regions compared to the quiet Sun: Livingston (1982), analysing spectra obtained with the FTS at KPNO and Cavallini et al. (1984), analysing the Fabry-Perot measurements made at Arcetri Observatory, find a red-shift; Kaisig and Schröter (1983) find a blue-shift. These authors interpret the blue-shift as a penetration of convective flux into higher photospheric levels in active regions, while the former interpret the measured red-shift as a retardation or an inhibition of convection.

In the quiet network, at supergranular boundaries, the wings of three lines $(g=0)$ are red-shifted by between $75-200 \mathrm{~m} \mathrm{~s}^{-1}$ relative to the cell profiles. The cores are relatively unshifted (Miller et al., 1984). Such trends can result if granular convection is suppressed near the network flux tubes, so that there is little downflow in the vicinity of the flux tubes.

Accuracy of absolute measurements. The accuracy of Doppler-shift measurements is limited by uncertainties of the position of reference lines. Causes of reference line shifts are analysed, and solutions allowing an improvement of measurement accuracy are given by Balthasar et al. (1982) for telluric reference lines and by Koch and Wöhl (1984) for iodine reference lines.

\section{Theoretical models of the solar granulation}

We will restrict ourselves to those theoretical models of convection which are closely related to the solar granulation.

Nordlung (1982) develops a full three-dimensional numerical simulation of the solar granulation. He solves the hydrodynamic equations, including the radiative transfer equations, in the anelastic approximation; turbulent viscous terms are included in the equation of motion; the importance of spectral lines on the energy balance in the upper photosphere is stressed.

The results of these simulations show the main observed characteristics of the granulation (Dravins et al., 1981; Nordlung, 1984 b): granules surrounded by intergranular lanes; downward motion in the lanes of larger amplitude than the upward motion in the granules; granules increasing their horizontal dimension in time; larger granules breaking apart into smaller ones, which in turn grow and merge. Synthetic spectral lines calculated both for the solar disc center and for the Sun as a star are in good agreement with the widths, strengths and shapes of observed spectral lines. The spectacular fit of the simulations with observations makes Nordlung's tri-dimensional model very appealing, although it is inherent to the model not to be able to predict a turbulent cascade toward the smaller scales. In this model granules are driven by the buoyancy force, horizontal motions are driven by pressure gradients, and the centres of granules are cooled while they expand and brake. The model does not contain any arbitrary parameters: the typical amplitudes of velocity and temperature fluctuations depend only on effective temperature, surface gravity, and the chemical abundance.

Under a different approach (Narasimha and Antia, 1982), convective modes in the solar envelope are investigated in the frame of the mixing length theory. The structure of the convection zone calculated according to this theory is found to be consistent with the transport of convective flux by a linear superposition of statistically independent unstable convective modes, provided the effects of turbulent conductivity and viscosity are taken in to account. The resultant vertical velocity is in reasonable agreement with observed granular velocity. In a subsequent paper Antia et al. (1983) investigated the stability of linear convective modes in the solar envelope model by incorporating in a very crude manner the effects of turbulent pressure and eddy transport coefficients calculated in the mixing length approximation. It is demonstrated that for a reasonable choice of parameters there occur two peaks in the plot of growth rate versus horizontal wavenumber, whieh correspond to the observed features (horizontal size and lifetime) associated with the granulation and supergranulation.

\section{Variation of the properties of solar granulation over the activity cycle.}

A set of converging results of different origin have recently been published, indicating that the structure of the solar granulation varies over the solar cycle. 
The mean size of granules, either measured by the distance between the centres of granules, or by the number of granules per unit surface area, decreases with increasing activity (Macris and Rösch, 1983; Macris et al., 1984, Muller and Roudier, 1984). The granule-intergranular lane intensity ratio at $5200 \AA$ varies with time, being minimum (1.10) around the minimum of activity, and maximum (1.30) around activity maximum. All these measurements were made on high resolution photographs of the solar granulation performed with the $50 \mathrm{~cm}$ refractor at Pic du Midi Observatory.

The equivalent width of photospheric lines, measured with the $13.5 \mathrm{~m}$ spectrometer at Kitt Peak National Observatory, decreases by a factor ranging from 0 to $2.3 \%$ from 1976 to 1980 (Livingston and Holweger, 1982). The authors come to the conclusion that the weakenings are due to global variations of surface properties, namely a reduction of the temperature gradient through the low photosphere. Bisectors of strong iron lines in the full disk Fraunhofer spectrum, measured with the Fourier Transform Spectrometer at Kitt Peak National Observatory, are observed to diminish in curvature as the activity cycle proceeds from minimum (1976) to maximum (1979-1982). The implication is reduced convection on a global scale in response to an increase of total magnetic flux (Livingston et al. 1982; Livingston, 1983, 1984).

\section{References}

Alissandrakis, C.E, Macris, C.J., and Zacharidis, G. 1982, Solar Phys. 76, 129. Antia, H.M., Chitre, S.M., and Narasimha, D. 1983, M.N.R.A.S. 187, 23.

Balthasar, H. 1984, Solar Phys., 93, 219.

Balthasar, H., Thiele, U., and Wöhl, H. 1982, Astr. Ap. 114, 357.

Bässgen, M., and Deubner, F.L. 1982, Astr. Ap. 111, L1.

Brandt, P.N., and Schröter, E.H. 1982, Solar Phys. 79, 3.

Brandt, P.N., and Schröter, E.H. 1984, in Keil, 1984.

Bray, R.J., 1982, Solar Phys. 77, 299.

Bray, R.J., and Loughhead, R.E., 1984, Astr. Ap. 133, 409.

Buonaura, B. and Caccin, B., 1982, Astr. Ap. 111, 113.

Cavallini, F., Ceppatelli, G., and Righini, A. 1982, Astr. Ap. 109, 233.

Cavallini F., Ceppatelli, G., and Righini, A. 1984, in Keil, 1984.

Deubner, F.L. and Mattig, W. 1975, Astr. Ap., 45, 167.

Dravins, D. and Larsson B. 1984, in Keil, 1984.

Dravins, D., Lindegren, L., Nordlung, A. 1981, Astr. Ap., 96, 345.

Durrant, C.J., Kneer, F. and Maluck, G. 1981, Astr. Ap., 104, 211.

Durrant, C.J., Mattig, W., and Nesis, A. 1979, Solar Phys. 61, 251.

Durrant, C.J., Mattig, W., Nesis, A., and Schmidt, W. 1983, Astr. Ap., 123, 319.

Durrant, C.J. and Nesis, A. 1982, Astr. Ap. $111,272$.

Edmonds, F.N., and Jin-Chung Hsu 1983, Solar Phys. 83, 217.

Howard, R., Boyden, J.E., and LaBonte, B.J. 1980, Solar Phys. 66, 167.

Kaisig, M. and Durrant, C.J., 1982, Astr. Ap. 116, 332.

Kaisig, M. and Schröter, E.H. 1983, Astr. Ap. 117, 305.

Karpinsky, V.N. 1981, Soln. Dannye. 10, 88.

Keil, S. 1980, Ap. J. 207, 1024.

Keil, S. 1984 (ed), Small-Scale Dynamical Processes in the Solar and Stellar Atmospheres (Sacramento Peak Observatory: Sunspot, NM), in press.

Koch, A., and Wohl, H. 1984, Astr. Ap. 134, 134.

Kostick, R. 1983, Publication of the Academy of Science of Ukraine, 83, 63.

Lawrence, J.K. 1983, Solar Phys. 87, 1.

Lites, B.W. 1983, Solar Phys. 85, 193.

Livingston, W. 1983, in Solar and Stellar Magnetic Fields, J.O. Stenflo Ed.

Livingston, W. 1984, in Keil, 1984.

Livingston, W., and Holweger, H. 1982, Ap. J. 252, 375.

Macris, C.J., and Rösch, J. 1983, C.R. Acad. Sc. Paris, $296,265$.

Macris, C.J., and Rösch, J., and Roudier, T. 1984, in Kejl, 1984.

Marnılino, C. and Severino, G. 1981, Astr. Ap. 100, 191.

Mehltretter, J.P. 1978, Astr. Ap. 62, 311.

Miller, P., Foukal, P., and Keil, S. 1984, Solar Phys. $92,33$.

Muller, R. and Roudier, T. 1984 in Hydromagnetics of the Sun, Proc. 4th European Mtg. on Solar Physics, Noordwijk, in press. 
Narasimha, D., and Antia, H.M. 1982, Ap. J. 262, 358.

Nesis, A., Durrant, C.J. and Mattig, W., 1984, in Keil, 1984.

Nordlung, A. 1982, Astr. Ap. 107, 1.

Nordlung, A. 1984a, in Keil, 1984.

Nordlung, A. 1984b, in Keil, 1984.

Oda, N. 1984, Solar Phys. 93, 243.

Perfinenko, L.D., 1981, Soln. Dannye, 10, 101.

Pierce, A.K. 1984, Solar Phys. 00, 195.

Pierce, A.K. and Breckinridge, J.B., 1973, Kitt Peak Nat. Obs. Contrib. 559.

Pravdjuk, L.M. 1982, Soln. Dannye, 2, 102.

Ricort, G., Borgnino, J., and Aime C. 1982, Solar Phys. 75, 377.

Schmidt, W., Knölker, M., and Schröter, E.H. 1981, Solar Phys. 73, 217.

Von der Lühe, O. 1981, Astr. Ap. 101, 277.

Wiesmeier, A., and Durrant, C.J. 1981, Astr. Ap. 104, 207.

Wittman, A. 1981, Astr. Ap. 99, 90.

\section{DYNAMICS OF THE CHROMOSPHERE AND TRANSITION REGION \\ (R. Grant Athay)}

One of the more interesting aspects of the chromosphere-corona transition region is its tendency to exhibit large Doppler shifts. Both the non-thermal velocity component of line widths and the velocity displacement of line positions tend to maximize at temperatures near $10^{5} \mathrm{~K}$. The increase in velocity amplitudes with increasing temperatures below $10^{5} \mathrm{~K}$ is readily understood in terms of the increasing sound speed and decreasing densities associated with the outwardly increasing temperature. Why the observed velocity amplitudes should decrease at still higher temperatures is not at all clear, however, and it seems very likely that this phenomenon is indicative of fundamental differences in the dynamics of the upper transition region and corona from those in the lower transition region and chromosphere.

The possibility remains, of course, that the apparent velocity decrease at high temperatures is only partially a solar effect. At temperatures above $10^{5} \mathrm{~K}$, increases in temperature, in general, are associated with increasing amounts of radiating material as a result of the decreasing temperature gradients. The resulting increase in path length over which a given spectral line forms tends to blend together regions of differing Doppler shift. As a result, the lines are broadened in preference to overall wavelength displacements. The apparent decrease in Doppler shifts very likely is due partially to this effect. However, since no marked increase in the non-thermal component of line broadening has been observed for temperatures above $10^{5} \mathrm{~K}$, it is evident that the steady increase in velocity amplitude up to $10^{5} \mathrm{~K}$ does not continue at the same rate in to the $10^{6} \mathrm{~K}$ regime of the corona. Thus, at least part of the effect appears to be of solar origin.

A further unusual property of the temperature regime below about $10^{5} \mathrm{~K}$ is the tendency for the solar plasma to exhibit large scale systematic flows as well as both periodic and highly transient localized flows. This review concentrates on the observational aspects of these flows as reported from 1981 to mid-1984.

\section{Vertical Flow.}

A number of observers have continued to report systematic downflows observed in spectral lines formed in the lower transition region and chromosphere. Gebbie et al. (1981) report average downflow velocities for the quiet sun ranging from $1.4 \mathrm{~km} \mathrm{~s}^{-1}$ in C II to $4.2 \mathrm{~km} \mathrm{~s}^{-1}$ in C IV, which is consistent with momentum conservation. Roussel-Dupre and Shine (1982) find mean redshifts of $12 \mathrm{~km} \mathrm{~s}^{-1}$ in C IV and Si IV at disk center, and Dere (1982a) finds an average red shift of $5.4 \mathrm{~km} \mathrm{~s}^{-1}$ for the quiet sun in C IV.

In active regions, Feldman, Cohen and Doschek (1982) find mean red shifts ranging from 4 to $17 \mathrm{~km} \mathrm{~s}^{-1}$ in ions formed at different temperatures with the maximum occuring between $5 \times 10^{4}$ and $10^{5} \mathrm{~K}$ and decreasing to $2 \mathrm{~km} \mathrm{~s}^{-1}$ in lines of Si II, S II and C II formed in the upper chromosphere, and Brueckner (1981) finds downflows of $10-60 \mathrm{~km} \mathrm{~s}^{-1}$ in $\mathrm{C}$ IV.

Over sunspot umbrae the situation is less clear. Several authors (Brueckner 1981, Nicholas et al. 1982, Dere 1982b and Athay et al. 1982) have reported downflows in C IV generally exceeding those in the quiet sun. However, in subsequent studies Athay, Gurman and Henze (1983) found several spots with upflow and Gurman and Athay (1983) found from a study of 8 sunspots a small net average upflow. Also, Mein et al. (1982) found strong upflow in C IV in three sunspots; 